\title{
MicroRNA-107 inhibits proliferation of prostate cancer cells by targeting cyclin E1
}

\author{
X. ZHANG, K JIN, J. D. LUO, B. LIU, L. P. XIE* \\ Department of Urology, The First Affiliated Hospital, Zhejiang University School of Medicine, Hangzhou, Zhejiang, China \\ *Correspondence: xielp@zju.edu.cn
}

Received November 5, 2018 / Accepted March 5, 2019

\begin{abstract}
Previous studies have reported that miR-107 could be utilized as a potential peripheral biomarker in prostate cancer (PCa). However, the specific functions of miR-107 in prostate cancer and its relevant mechanisms are still unknown. The aim of this research was to investigate the cellular functions of miR-107 in PCa and reveal the relevant mechanisms. MicroRNA tailing quantitative real-time PCR (qRT-PCR) was adopted to measure the expression of miR-107 in PCa cell line DU145 and PC3, as well as in normal prostate cell line RWPE-1. The miR-107 expression pattern in PCa tissues and paired peritumoral tissues were determined by Chromogenic In Situ Hybridization (CISH). Cell viability, colony formation, flow cytometry cell cycle and apoptosis, wound healing and Transwell migration assays were performed to study the miR-107 functions in PCa cells. Further, qRT-PCR, western blot analysis and dual-luciferase reporter assays were conducted to verify the target of miR-107 in PCa. Results demonstrated that miR-107 was downregulated in PCa cells and tissues in comparison with normal prostate cells and peritumoral tissues, and the over-expression of miR-107 suppressed proliferation and induced G1/S arrest of PCa cells but had no effects on apoptosis or cell motility of PCa cells. MiR-107 was found to target cyclin E1 (CCNE1) in PCa cells by directly binding to its 3'-UTR. In conclusion, miR-107 could be a potential tumor suppressor in $\mathrm{PCa}$, and the restoration of miR-107 might provide a new therapeutic option for PCa.
\end{abstract}

Key words: prostate cancer, proliferation, microRNA-107, CCNE1

Prostate cancer $(\mathrm{PCa})$ is the second most occurring cancer and the fifth leading cause of cancer-related deaths in men worldwide [1]. Although the combination of modern surgery, radiotherapy and androgen-deprivation therapy (ADT) could remarkably improve the prognosis of $\mathrm{PCa}$ patients, the progression from androgen-sensitive prostate cancer to castration-resistant prostate cancer (CRPC) remains formidable and poses considerable challenges to PCa treatment. Present developments of multitudinous regimens for $\mathrm{PCa}$ are ongoing, among which RNAi therapies have very promising prospects.

MicroRNA (miRNA) is a 21-23 nucleotide-length endogenous non-coding RNA, which usually functions through its binding with the 3'-untranslated regions (3'-UTRs) of its target mRNAs. This leads to their degradation or transcriptional silencing, and therefore makes miRNA to be key players in multiple biological processes, such as development, differentiation, metabolism, immune response, oncogenesis, and so forth [2, 3]. High efficacy of RNAi therapies has spurred explorations of novel approaches to improve miRNA/siRNA delivery, for example engineered lipid nanoparticles have been developed to overcome the fragility of miRNA/siRNA in the presence of abundant RNases in human body and promote the target specificity $[4,5]$. Besides, clinical trials of RNAi therapies have been launched $[6,7]$. Despite the rapid progress in the field of RNAi therapies, fundamental work on identification of new therapeutic miRNAs in human diseases is still important. Last year our laboratory discovered two miRNAs, miR-493-5p and miR-493-3p, as tumor suppressors in $\mathrm{PCa}$, which participated in the regulation of proliferation, cell cycle, apoptosis, migration and invasion of PCa by targeting different oncogenes $[8,9]$.

MiR-107 is a conserved miRNA transcribed from the introns of vertebrate pantothenate kinase 1 gene (PANK1) [10]. It has been implicated in various physiological and pathological processes, including lipid metabolism, tumor angiogenesis, pathogenesis of diabetes and Alzheimer's disease, etc. [11-15]. However, the functions of miR-107, particularly in prostate cancer, have not been systematically investigated. In this current work, it was demonstrated that miR-107 was downregulated in PCa cell lines and tissues in contrast to normal prostate cell line and tissues, and it suppressed the proliferation of PCa cells via targeting cyclin E1 (CCNE1). 


\section{Materials and methods}

Cell culture. PCa cell lines DU145 and PC3, normal prostate cell line RWPE-1 and HEK 293T cell line were purchased from Stem Cell Bank, Chinese Academy of Science. DU145 cell line was derived from a PCa metastatic brain lesion of a 69-year-old white male [16], and PC3 cell line was derived from PCa bone metastasis of a 62-yearold white male [17]. RWPE-1 cell line was derived from the prostate tissue of a healthy 54-year-old white male [18]. The PCa and HEK 293T cells were cultured in minimal essential medium (MEM, Corning, USA) and Dulbecco's modified Eagle's medium (DMEM, Corning, USA) respectively, supplemented with $10 \%$ fetal bovine serum (FBS, Biological Industries, Israel), while RWPE-1 cells were cultured in keratinocyte serum free medium (K-SFM, Gibco, USA). All cells were cultured in $37^{\circ} \mathrm{C}$ thermostatic atmospheres with $5 \% \mathrm{CO}_{2}$.

Transient transfection of microRNA mimic, microRNA inhibitor,and small interfering RNA (siRNA). All microRNA mimics, inhibitors and siRNA duplexes were synthesized by GenePharma (Shanghai, China). MicroRNA107 mimic was employed for the gain-of-function studies and the siRNA targeting CCNE1 mRNA was designed by GenePharma for knocking-down CCNE1 in PCa cells. Single-stranded microRNA-107 inhibitor could bind to and repress intrinsic microRNA-107 and was utilized for the CCNE1 rescue experiments. Negative control microRNA duplex was designated $\mathrm{NC}$ and had no biological effects in human cells. The sequences of all the RNA oligonucleotides are shown in Table 1. Lipofectamine 2000 (Invitrogen, USA) was used for transient transfection based on the manufacturer's guidelines.

Chromogenic in Situ Hybridization (CISH) staining. A 5'-DIG (digoxigenin) and 3'-DIG double-labeled oligonucle- otide miRNA probe (Servicebio, Wuhan, China) was used for the detection of miR-107 in PCa tissue microarray (TMA) which contained 20 cases of PCa samples with paired peritumoral tissues. TMA was purchased from Servicebio, Wuhan, China. Paraffin tissue slide was deparaffinized and digested with proteinase $\mathrm{K}$ for 5 minutes. Subsequently the slide was pre-hybridized in the hybridization solution at $50^{\circ} \mathrm{C}$ for 1 hour. Tissue slide was hybridized for 2 days in the presence of $10 \mathrm{ng}$ miR-107 probes at $4{ }^{\circ} \mathrm{C}$ and was washed stringently in $50^{\circ} \mathrm{C}$ for 20 minutes. The DIG double-labeled miR-107 probe was detected with sequential incubation with HRP-Conjugated IgG Fraction Anti-DIG (Jackson ImmunoResearch, USA) for 1 hour at room temperature and DAB development for 30 minutes. Finally, tissue slide was counterstained with hematoxylin, dehydrated and coverslipped. The strength of miR-107 positivity was semi-quantified by evaluating both the proportion and intensity of the miR-107 positive cells.

Cell viability assay. $P$ Ca cells were seeded in 96 -well plates at the density of $4 \times 10^{3}$ cells per well. After overnight cultivation, miR-107 mimic/CCNE1 siRNA of different concentrations ranging from $0 \mathrm{nM}$ to $75 \mathrm{nM}$ were transfected into PCa cells. The concentration of NC for transfection was $75 \mathrm{nM}$. Twenty-four, 48 and 72 hours after transfection, the medium was removed, and the Cell Counting Kit 8 (CCK8, Dojindo, Japan) reagent dissolved in nine volumes of complete MEM was added to each well and incubated in $37^{\circ} \mathrm{C}$ for 1 hour. The absorbance at wavelength of $450 \mathrm{~nm}$ for each well was measured by Elx 800 absorbance reader (BioTek Instruments, USA).

Colony formation assay. PCa cells transfected with miR-107 mimic/CCNE1 siRNA were harvested 48 hours after transfection and re-seeded in 6-well plates with 500 cells per well. The cells were again cultured under normal conditions. 10 days later the cells were washed with PBS, fixed with absolute methanol for 10 minutes and stained with $0.1 \%$

Table 1. The oligonucleotides used in this study.

\begin{tabular}{|c|c|}
\hline Name $^{a}$ & Sequences $\left(5^{\prime}-3^{\prime}\right)^{b}$ \\
\hline miR-107 mimic (sense) & AGCAGCAUUGUACAGGGCUAUCA \\
\hline NC (sense) & UUCUCCGAACGUGUCACGU \\
\hline miR-107 inhibitor & UGAUAGCCCUGUACAAUGCUGCU \\
\hline inhibitor NC & CAGUACUUUUGUGUAGUACAA \\
\hline miR-107 F & AGCAGCATTGTACAGGGCTATCA \\
\hline miR-107 probe ${ }^{c}$ & TGATAGCCCTGTACAATGCTGCT \\
\hline CCNE1 F & AAGGAGCGGGACACCATGA \\
\hline CCNE1 R & ACGGTCACGTTTGCCTTCC \\
\hline GAPDH F & CTGGGCTACACTGAGCACC \\
\hline GAPDH R & AAGTGGTCGTTGAGGGCAATG \\
\hline CCNE1 UTR Wt F & CGACACCAGTGCGTGCTCCCGATGCTGCTATGGAAGGTGCTACTTGACCTG \\
\hline CCNE1 UTR Wt R & TCGACAGGTCAAGTAGCACCTTCCATAGCAGCATCGGGAGCACGCACTGGTGTCGAGCT \\
\hline CCNE1 UTR Mut F & CGACACCAGTGCGTGCTCCCGTACGACGAATGGAAGGTGCTACTTGACCTG \\
\hline CCNE1 UTR Mut R & TCGACAGGTCAAGTAGCACCTTCCATTCGTCGTACGGGAGCACGCACTGGTGTCGAGCT \\
\hline CCNE1 siRNA (sense) & CCUGGAUGUUGACUGCCUU \\
\hline
\end{tabular}

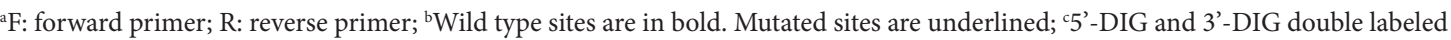


crystal violet (Solarbio, Beijing, China) for 15 minutes. The colonies with a diameter of over $1 \mathrm{~mm}$ were counted.

Flow cytometry cell cycle assay. PCa cells transfected with miR-107 mimic/CCNE1 siRNA were harvested 48 hours after transfection and fixed in $75 \%$ ethanol at $-20^{\circ} \mathrm{C}$ overnight. Then, the cells were collected, washed with PBS and stained with propidium iodide (Liankebio, Hangzhou, China) for 30 minutes. Cell cycle assay was achieved by FACSCantoII flow cytometry (BD, USA) and the cell cycle distribution was analyzed by ModFit 4.0 software.

Flow cytometry apoptosis assay. Seventy-two hours after miR-107 mimic transfection, all PCa cells (including cells in the medium) were harvested and stained with Annexin V-APC Apoptosis Kit (Liankebio, Hangzhou, China) according to the manufacturer's instructions. Apoptosis assay was achieved by FACSCantoII flow cytometry and the apoptotic status was analyzed by FlowJo 10.0 software.

Wound healing assay. PCa cells were seeded in 6-well plates to make sure that the cells reached $100 \%$ confluence 24 hours after miR-107 mimic transfection. The wound of '+' shape was made in the center of the well with a $200 \mu$ l pipette tip and medium was replaced with serum-free MEM. Photos were taken 24 hours and 48 hours after the wounding and cells were cultured in serum-free MEM during the whole procedure.

Transwell migration assay. Twenty-four hours after miR-107 mimic transfection, PCa cells were harvested and resuspended in serum-free MEM, and $8 \times 10^{4}$ cells were re-seeded on Millicell 24-Well Hanging Inserts (Millipore, Germany). The hanging inserts containing PCa cells were mounted in 24-well plates with $700 \mu$ l complete MEM per well and placed back to standard culture environment. Twenty-four hours later the cells on the upside of the hanging inserts were removed, and the cells on the downside were fixed with absolute methanol for 10 minutes and stained with $0.1 \%$ crystal violet (Solarbio, Beijing, China) for 15 minutes. Finally, the membranes on which the cells attached were mounted on slides and sealed in neutral balsam, and then observed microscopically at a magnification of $200 \times$.

RNA extraction and quantitative real-time PCR (qRT-PCR). Total RNA of PCa cells was extracted with RNAiso Plus (Takara, Japan) 48 hours after transfection. For ordinary qRT-PCR, mRNA was reversely transcribed into cDNA with PrimeScript RT Master Mix (Takara, Japan), and for miRNA tailing qRT-PCR, miRNA was converted into tailed cDNA with Mir-X MiRNA First Strand Synthesis Kit (Takara, Japan). TB Green Premix Ex Taq II (Takara, Japan) was used for the amplification of both mRNA and miRNA, and the quantification was accomplished by CFX96 RealTime PCR Detection System (Bio-Rad, U.S.A.). All of the PCR-associated procedures were performed according to the protocols prescribed on the corresponding reagents or kits. The sequences of all the primers are shown in Table 1 . The results were analyzed by $2^{-\Delta \Delta \mathrm{Ct}}$ method.

Western blot analysis. PCa cells were collected 48 hours after transfection and lysed with RIPA lysis buffer (Servicebio,
Wuhan, China) supplemented with protease and phosphatase inhibitor (Pierce Mini Tablets, Thermo Scientific, USA) on ice for 30 minutes. BCA Protein Assay Kit (Beyotime, Shanghai, China) was used to calculate the total protein concentration. Protein samples were electrophoresed and separated in $4-20 \%$ polyacrylamide gels (GenScript, Nanjing, China) with $30 \mu$ g protein per well and transferred to polyvinylidene fluoride (PVDF) membranes (Millipore, Germany). The PVDF membranes were blocked with 5\% non-fat milk for 2 hours, and incubated with primary antibodies overnight, including anti-GAPDH, anti-CDK2 (Proteintech, USA), and anti-CCNE1 (Cell Signaling Technology, USA) antibodies. Thereafter, PVDF membranes were washed three times in TBS-T solution and incubated with horseradish peroxidase (HRP)-conjugated secondary antibodies (Proteintech, USA) for 1 hour. The blots were detected by Enhanced Chemiluminescence Kit (FDbio, Hangzhou, China).

Immunohistochemistry (IHC) staining. PCa TMA was stained with IHC to assess the expression of CCNE1. TMA was purchased from Xinchao Biotech, Shanghai, China, which contained 36 cases of PCa samples with paired peritumoral tissues. Tissue section was dewaxed and rehydrated through graded alcohols. Antigen retrieval was performed by heating the microarray in $0.01 \mathrm{M}$ citrate buffer $(\mathrm{pH}=6.0)$ and $3 \%$ hydrogen peroxide solution was used to block the endogenous peroxidase activity. The section was then incubated with anti-CCNE1 (Proteintech, USA) overnight at $4^{\circ} \mathrm{C}$. After washing, the microarray was incubated with HRP-conjugated secondary antibodies (Proteintech, USA) for 1 hour at room temperature and developed by DAB. Finally, tissue section was counterstained with hematoxylin, dehydrated through graded alcohols, cleared in xylene and coverslipped in neutral balsam. The strength of CCNE1 positivity was semiquantified by evaluating both the proportion and intensity of the CCNE1 positive cells.

Dual-luciferase reporter assay. The segments of CCNE1 3'-UTR containing either the predicted wild type (Wt) or the mutated type (Mut) miR-107 binding site were synthesized as sense and antisense oligomers by Sangon (Shanghai, China), annealed and cloned into dual-luciferase vector pmirGLO (Promega, USA) between the SacI and SalI restriction sites downstream of the firefly luciferase gene. Then Escherichia coli (E. coli) DH5a competent cells (Takara, Japan) were transformed with the reconstructed vectors by heat shock according to the manufacturer's instructions. After transformation, DH5a competent cells were plated on solid LuriaBertani (LB) agarose medium supplemented with ampicillin and cultivated in $37^{\circ} \mathrm{C}$ for 16 hours. When bacterial colonies were visible, single colonies were picked out and amplified in ampicillin-supplemented liquid $\mathrm{LB}$ medium in $37^{\circ} \mathrm{C}$ overnight. The reconstructed vectors within $\mathrm{DH} 5 \mathrm{a}$ cells were extracted with E.Z.N.A Endo-Free Plasmid Mini Kit (Omega Bio-Tek, UK). Finally, the correct insertion of the wild type or mutated type 3'-UTR segments into dual-luciferase vector was confirmed by second-generation sequencing. 
HEK 293T cells were plated in 48-well plates and co-transfected with $50 \mathrm{nM}$ miR-107 mimic/NC and $50 \mathrm{ng}$ dual-luciferase vector. 48 hours after co-transfection, E1910 dual-luciferase reporter assay system (Promega, USA) was adopted for measuring the activities of firefly luciferase (luc2) and Renilla luciferase (Rluc) based on the manufacturer's instructions. The relative luciferase activity was presented as the ratio of firefly luciferase (luc2) activity to Renilla luciferase (Rluc) activity.

CCNE1 rescue experiments. After overnight cultivation, CCNE1 siRNA and miR-107 inhibitor/inhibitor NC were co-transfected into PCa cells. Fourty-eight hours after transfection, PCa cells were harvested for cell cycle analysis, while western blot analysis of CCNE1 expression was performed 72 hours after transfection.

Oncomine database analysis. The analysis of CCNE1 expression pattern between normal prostate and PCa tissues was carried out using Oncomine database (https:// www.oncomine.org/). Its filter indexes were set as follows: primary filters were set as differential analysis (cancer versus normal tissue) and prostate cancer type. Dataset filters were set as mRNA data type, and the search pattern was set as CCNE1 gene. The differential expression of CCNE1 mRNA between normal prostate and PCa tissues was then displayed, alongside with detailed information such as sample source, sample size, $\mathrm{p}$-value, fold of change and statistical box plots.

Statistical analysis. The data were presented as the mean \pm S.D., Kolmogorov-Smirnov test was used to gauge the normality of the data distribution in each group. Differences between two normal distribution groups were analyzed with Student's t-test, while differences among two or more normal distribution groups were analyzed with ANOVA test. Differences between two paired abnormal distribution groups were analyzed with Wilcoxon sign-rank test, while differences among non-paired abnormal distribution groups were analyzed with non-parametric rank-sum test.

\section{Results}

MiR-107 is downregulated in PCa cell lines and tissues. In order to explore the expression pattern of miR-107 in PCa and normal prostate cells and tissues, microRNA tailing qRT-PCR was adopted to measure the miR-107 expression in
A

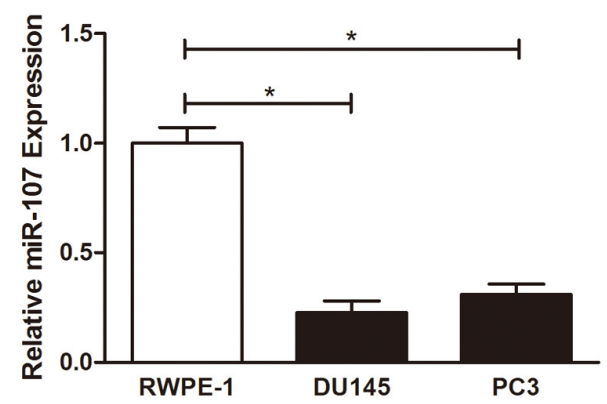

C

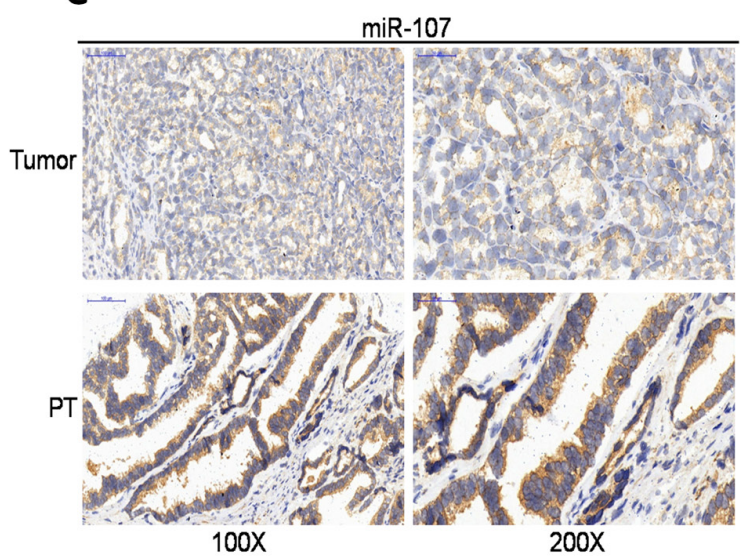

B

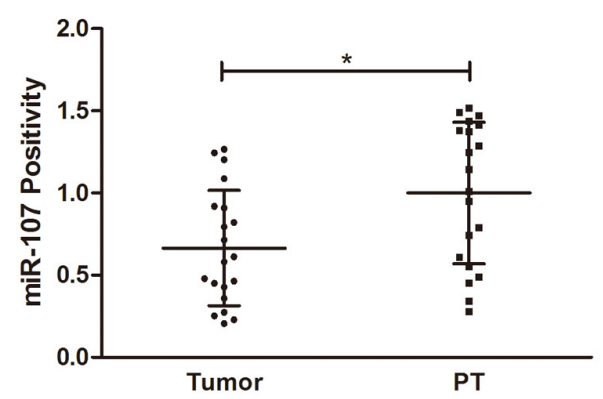

D

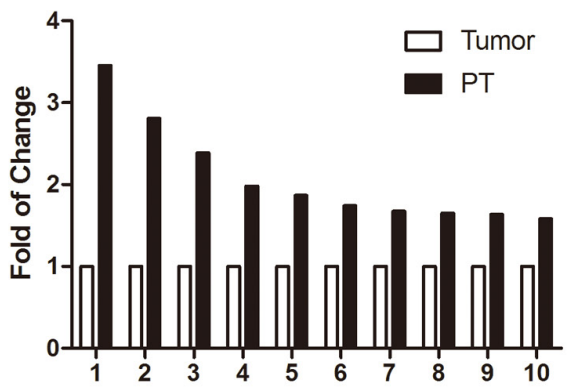

Figure 1. The expression pattern of miR-107 in PCa and normal prostate cells and tissues. A) MiR-107 tailing qRT-PCR. MiR-107 was downregulated sharply in PCa cell lines DU145 and PC3 compared with normal prostate cell line RWPE-1. B) Statistical analysis of miR-107 CISH staining in PCa TMA. MiR-107 expression was notably lower in PCa tissues than in peritumoral tissues (PT). C) Representative CISH staining of miR-107 in PCa TMA. D) The relative expression of miR-107 in 10 pairs of PCa tissues presented as the fold of change of miR-107 referring to the corresponding peritumoral tissues. Error bars represent S.D. from three independent experiments. ${ }^{*} \mathrm{p}<0.05$ 
PCa cell lines DU145 and PC3, and in normal prostate cell line RWPE-1. In comparison with PCa cell lines, miR-107 expression increased about four-fold in normal prostate cell line (Figure 1A). CISH experiment demonstrated that miR-107 was located in the cytoplasm of PCa cells, and similarly, the expression of miR-107 in PCa tissues was notably lower than in peritumoral tissues (Figures 1B-1D).

Overexpression of $\mathrm{miR}-107$ suppresses the proliferation of PCa cells. In the light of the low background of miR-107 in $\mathrm{PCa}$, gain-of-function strategies were employed to study how miR-107 influenced the behaviors of PCa cell lines DU145 and PC3. Intracellular overexpression of miR-107 could suppress the proliferation of PCa cells, as measured by CCK8 assay and colony formation assay. As shown in Figure 2A, the viabilities of DU145 and PC3 cells transfected with miR-107 mimic decreased evidently, and their colony-forming abilities were inhibited as well (Figure 2B).

Overexpression of miR-107 induces G1/S arrest of PCa cells. Since cell cycle progression is closely associated with cell proliferation, flow cytometry cell cycle assay was performed. The results showed that intracellular overexpression of miR-107 inhibited G1/S transition of DU145 cells, with an elevated proportion of cells in G1 phase and a reduced proportion of cells in S phase (Figure 3A). For PC3 cells, intracellular overexpression of miR-107 significantly increased the proportion of cells in G1 phase and decreased the proportion of cells in G2 phase, with no significant changes of the proportion of S phase (Figure 3A).

Upregulation of miR-107 does not induce apoptosis of PCa cells. To further clarify the anti-proliferative mechanisms of miR-107 in PCa, the apoptotic rates of PCa cells after intracellular upregulation of miR-107 were determined by flow cytometry apoptosis assay. No significant differences were observed regarding the apoptotic rates of miR-107 mimic-transfected PCa cells compared with NC-treated cells (Figure 3B). This indicated that the anti-proliferative mechanisms of miR-107 in PCa were independent of apoptosis signaling pathways.

MiR-107 has no effect on PCa cell motility. A study on how miR-107 could affect the phenotypes related to $\mathrm{PCa}$ cell motility was also carried out. In the Transwell migration assay, PCa cells in serum-free medium of the upper chamber migrated to the lower chamber containing a complete medium, due to the chemotactic attraction gener-
A

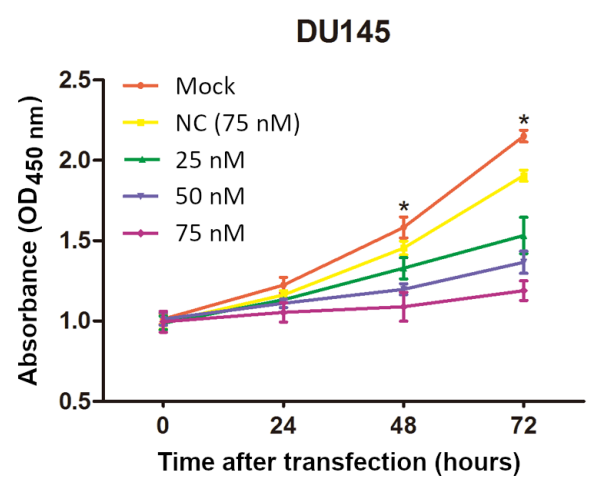

PC3

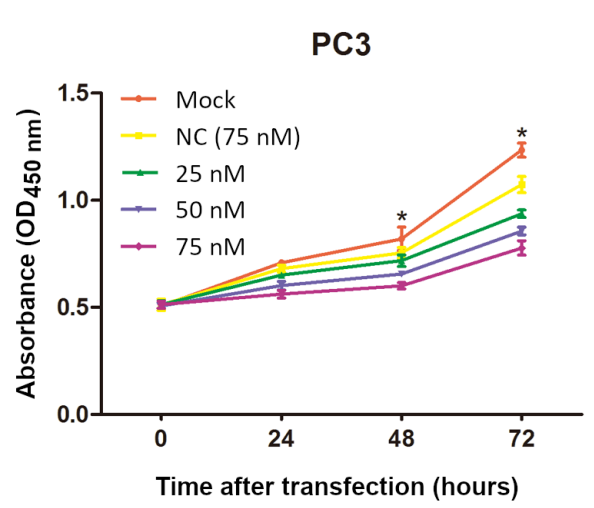

B

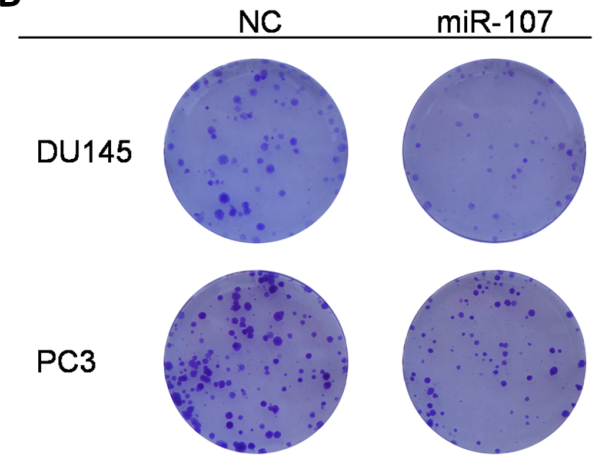

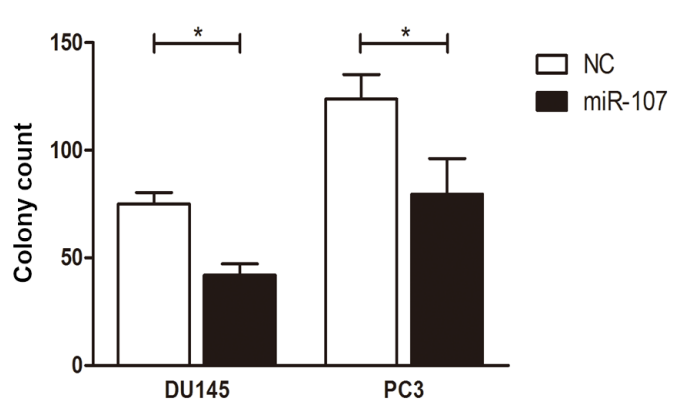

Figure 2. Overexpression of miR-107 suppresses the proliferation of PCa cells. A) Growth curves of PCa cells transfected with miR-107 mimic in serial concentrations. MiR-107 mimic-transfected PCa cells showed decreased viabilities compared with NC-treated PCa cells. Error bars represent S.D. from three independent experiments. B) Colony formation assay. The colony formation rates of PCa cells transfected with miR-107 mimic were reduced compared with NC-treated PCa cells. Error bars represent S.D. from three independent triplicate experiments. ${ }^{*} \mathbf{p}<0.05$ 
A
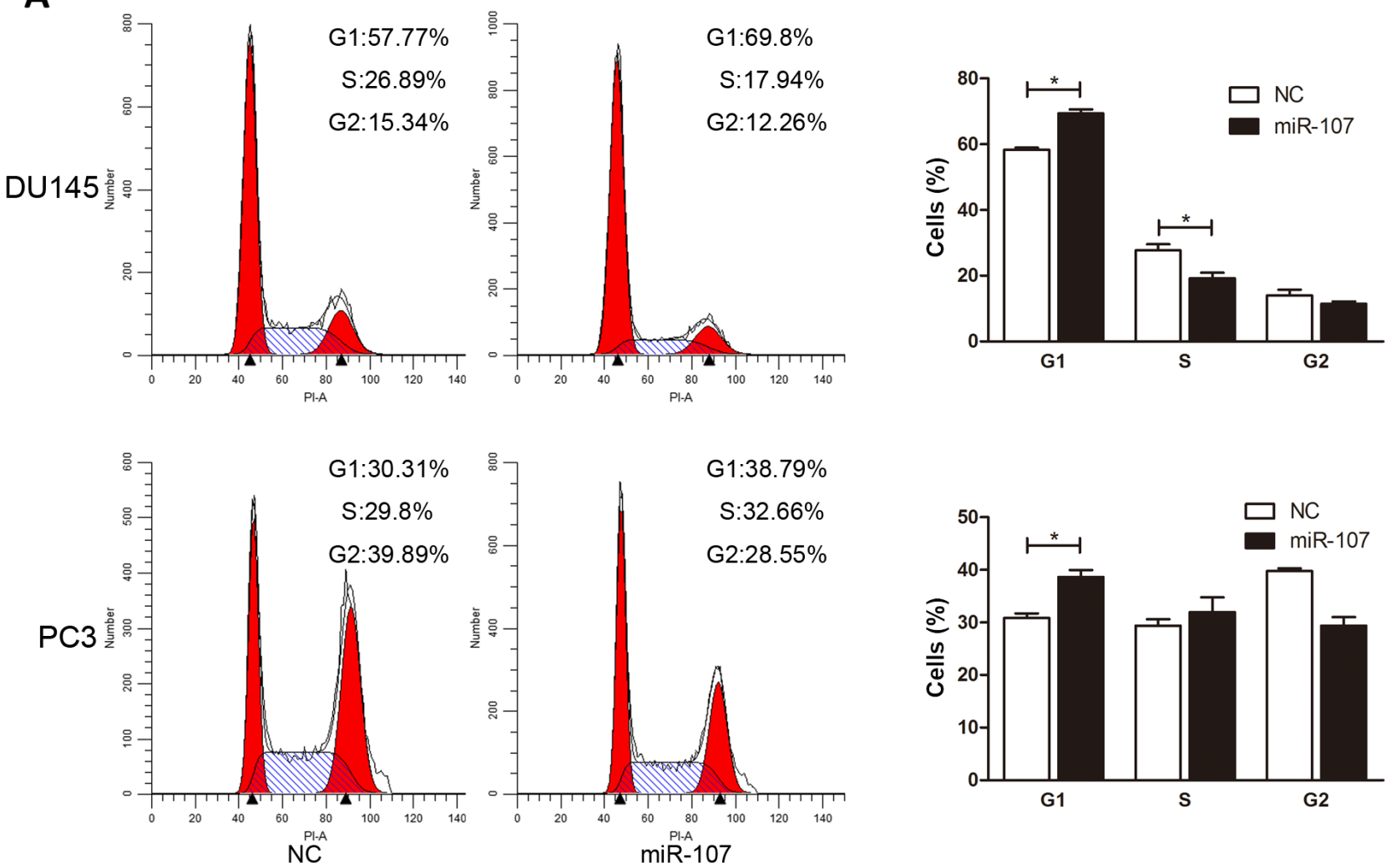

B
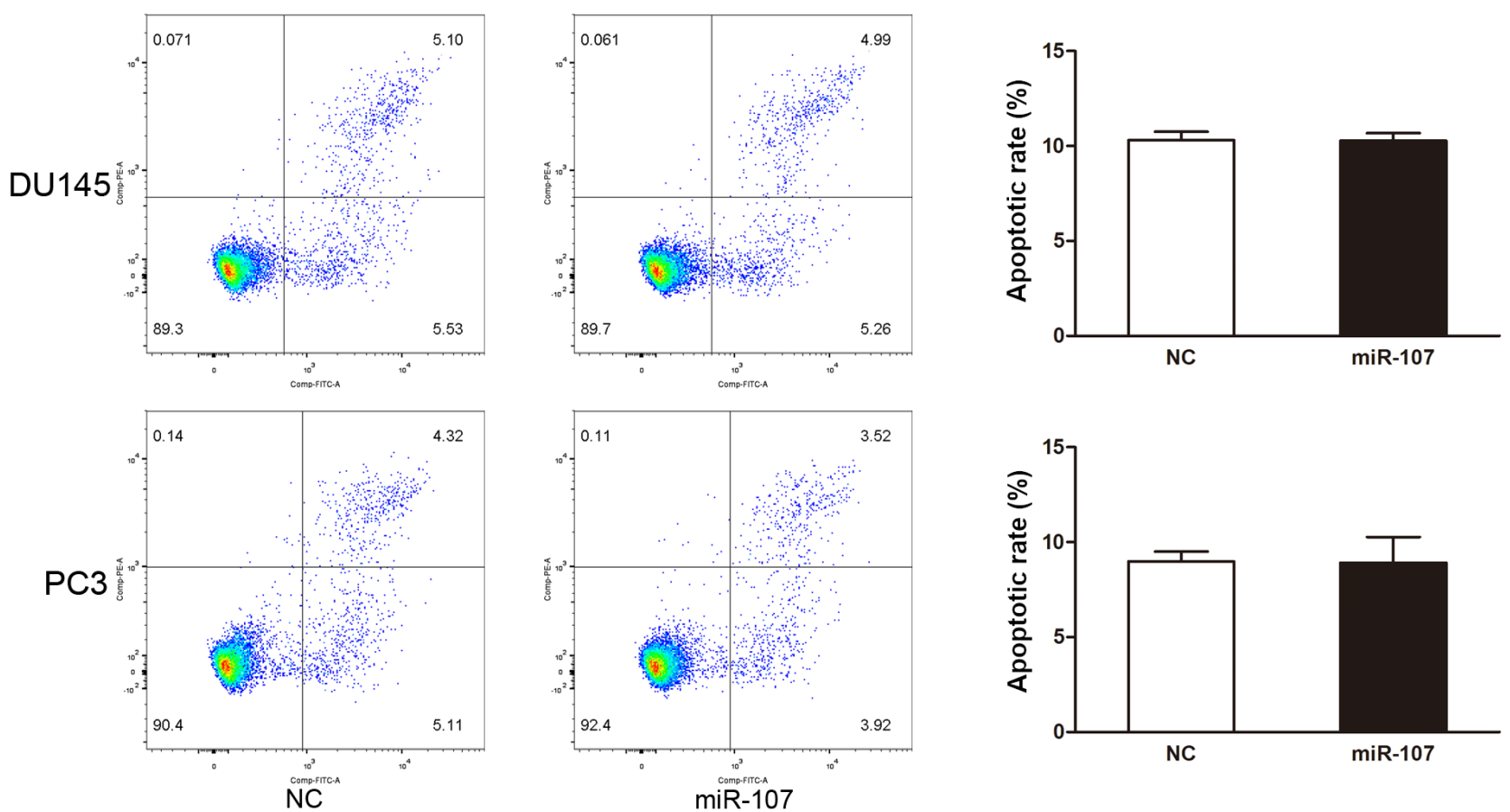

Figure 3. Flow cytometry cell cycle assay and flow cytometry apoptosis assay. A) Overexpression of miR-107 induced G1/S arrest of PCa cells. B) The apoptotic rates of miR-107 mimic-transfected PCa cells were not altered compared with NC-treated PCa cells. Error bars represent S.D. from three independent experiments. ${ }^{*} \mathbf{p}<0.05$ 
ated by serum gradient. It was found that miR-107 was non-functional with respect to PCa cell motility, as proved by the unchanged wound healing rates (Figure 4A) and the transwell migratory rates (Figure $4 \mathrm{~B}$ ) of miR-107 mimictransfected PCa cells as compared to NC-treated PCa cells.

MiR-107 downregulates the expression of CCNE1 by directly targeting its 3'-UTR. Based on previous cell function assays, the microRNA databases were analyzed for the possible targets of miR-107 in PCa cells. The functions of possible target proteins had to coincide with that of miR-107, and it was predicted that cyclin E1 (CCNE1) was directly targeted by miR-107. After PCa cells were transfected with miR-107 mimic, the expression of CCNE1 decreased significantly at both mRNA and protein levels (Figures 5A, 5B). Western blot analysis demonstrated that the expression of CCNE1 was higher in PCa cell line DU145 and PC 3 compared with normal prostate cell line RWPE-1 (Figure 5G). In addition, analyses of CCNE1 expression pattern using Oncomine database and CCNE1 IHC staining in PCa TMA also indicated its upregulation in PCa tissues compared with normal prostate tissues (Figures 5E, 5F and $5 \mathrm{H})$.

The direct targeting of CCNE1 by miR-107 was confirmed by dual-luciferase reporter assay. The segments of CCNE1 3'-UTR containing either predicted wild type
(Wt) or mutated type (Mut) miR-107 binding site were inserted into dual-luciferase vector pmirGLO (sequences shown in Figure 5C and Table 1) with the segments located downstream of the firefly luciferase gene. Targeting of 3'-UTR segment downstream of the firefly luciferase gene by miR-107 downregulated the expression of the firefly luciferase (luc2) while the expression of the Renilla luciferase (Rluc) was unchanged, which decreased the relative luciferase activity. Results demonstrated that compared with NC, miR-107 mimic downregulated the relative luciferase activities of Wt vector-transfected HEK 293T cells, while the Mut vector-transfected HEK 293T cells which were co-transfected with either miR-107 mimic or NC, showed no significant differences in their relative luciferase activities, which supported the direct targeting of CCNE1 3'-UTR by miR-107 (Figure 5D).

Knocking down of CCNE1 mimics the effects of miR-107 in PCa cells and could be reversed by miR-107 inhibitor. As CCNE1 was validated to be targeted by miR-107, cell proliferation assays and CCNE1 rescue experiments were conducted to further elucidate the association between miR-107 and CCNE1 in PCa. Results showed that intracellular knocking-down of CCNE1 by synthetic siRNA (Figure 6A) could reproduce the anti-proliferative and cell cycle-obstructive effects of miR-107 in PCa cells
A
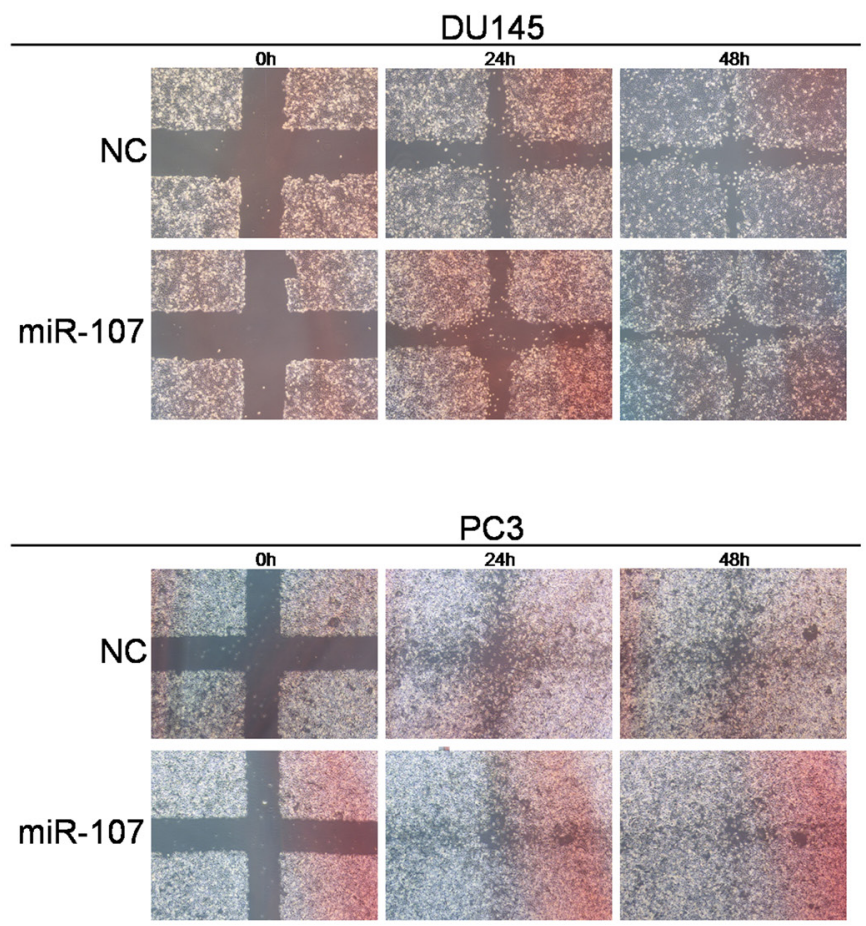

B
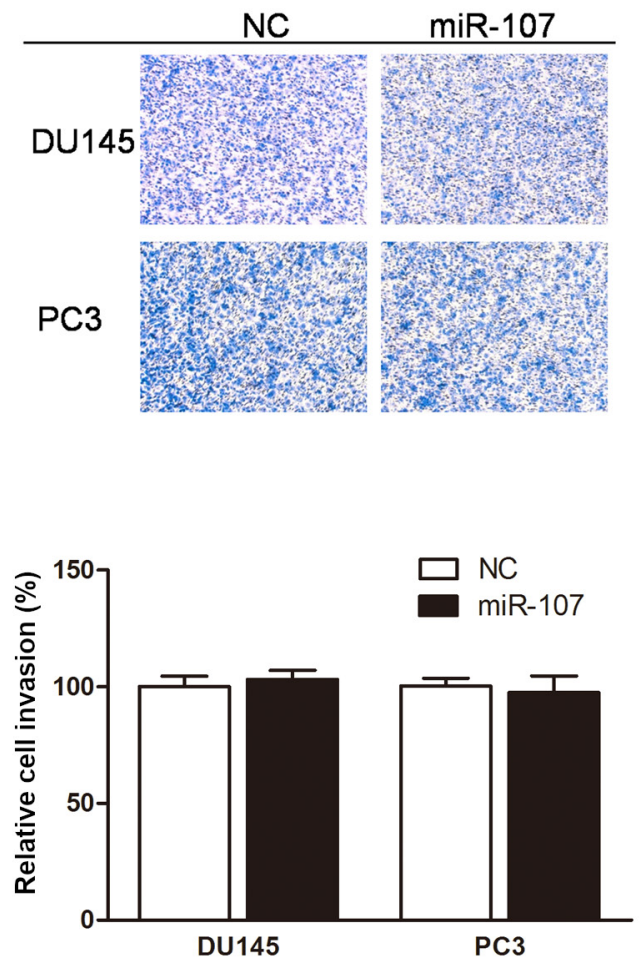

Figure 4. Wound healing assay and Transwell migration assay. A) The wound healing rates and B) the transwell migratory rates of miR-107 mimictransfected PCa cells were unchanged compared with NC-treated PCa cells. Error bars represent S.D. from three independent experiments. 
A

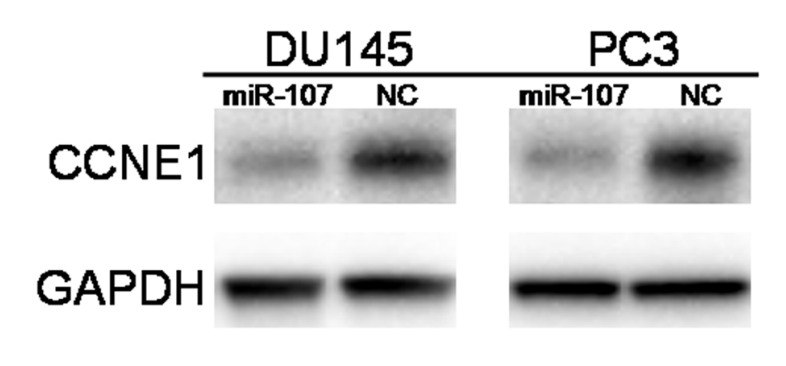

C

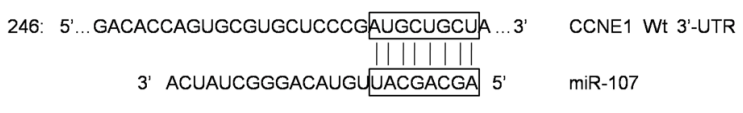

246: 5'...GACACCAGUGCGUGCUCCCG JACGACGAT ... 3' CCNE1 Mut 3'-UTR

D

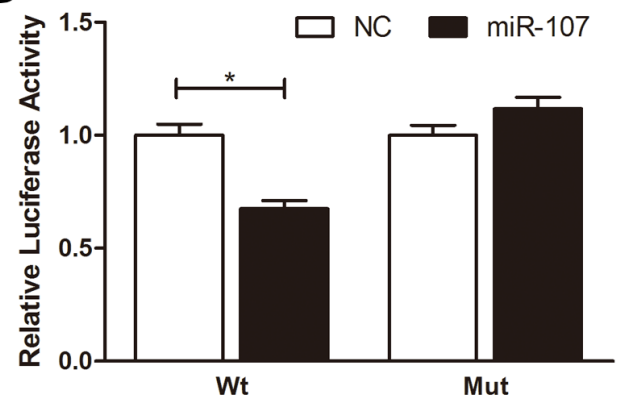

$\mathbf{F}$

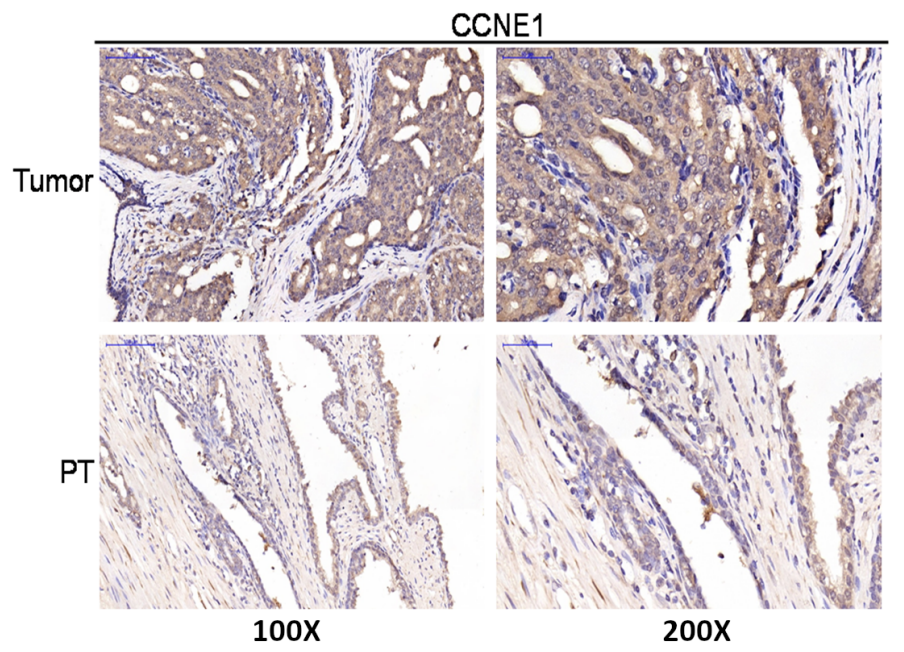

B

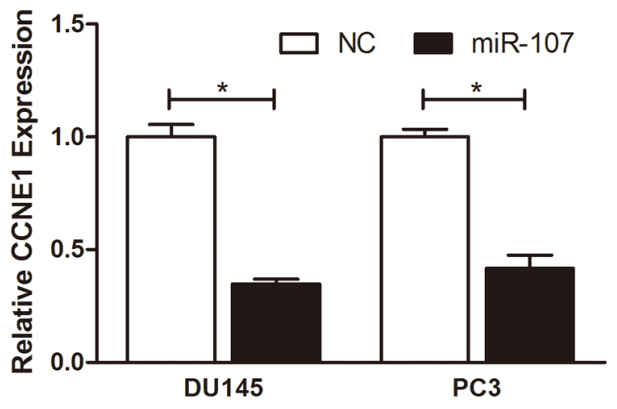

$\mathrm{E}$

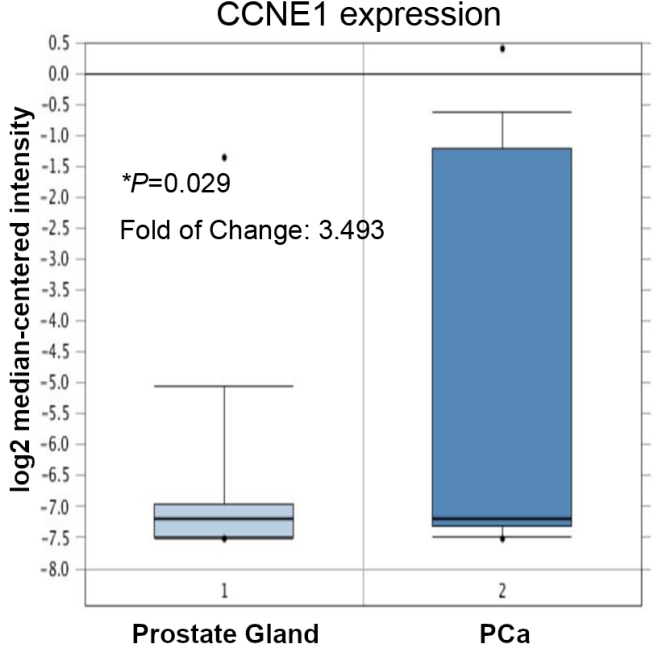

G

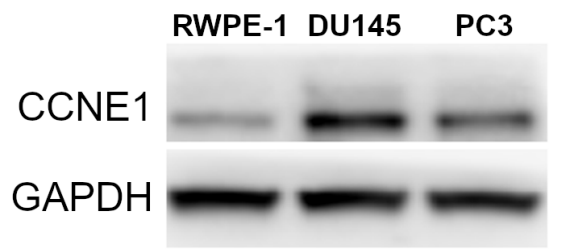

H

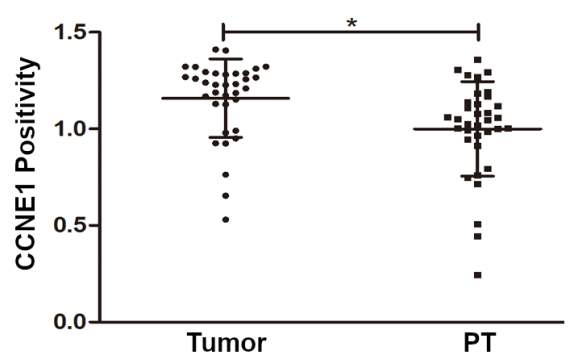

Figure 5. MiR-107 directly targets CCNE1 3'-UTR. A and B) qRT-PCR and western blot analysis. MiR-107 downregulated the expression of CCNE1 at mRNA and protein levels in PCa cells. C) Segments of CCNE1 3'-UTR containing either the predicted wild type (Wt) or the mutated type (Mut) miR107 binding site. D) Dual-luciferase reporter assay. Compared with NC, miR-107 mimic downregulated the relative luciferase activities of Wt vectortransfected HEK 293T cells, but not Mut vector-transfected HEK 293T cells. E) CCNE1 mRNA is upregulated in PCa compared with normal prostate tissues (data downloaded from Oncomine). The fold of change of CCNE1 mRNA expression in PCa compared with normal prostate tissues is 3.493. ${ }^{*} \mathrm{p}=\mathbf{0 . 0 2 9}$. The results are from Luo's microarray data sets, which include 15 cases of PCa and 15 cases of normal prostate tissues. F) Representative IHC staining of CCNE1 in PCa TMA. G) Western blot analysis. CCNE1 protein was upregulated in PCa cell lines DU145 and PC3 compared with normal prostate cell line RWPE-1. H) Statistical analysis of CCNE1 IHC staining in PCa TMA. CCNE1 expression was notably higher in PCa tissues than in peritumoral tissues (PT). Error bars represent S.D. from three independent experiments. ${ }^{*} \mathrm{p}<0.05$ 
A

CCNE1

GAPDH

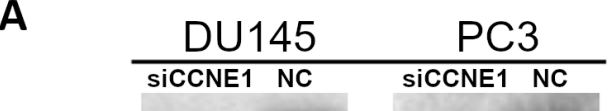

\section{1}

$-\cos 20$

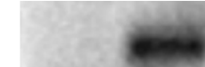

$\mathrm{H}=$

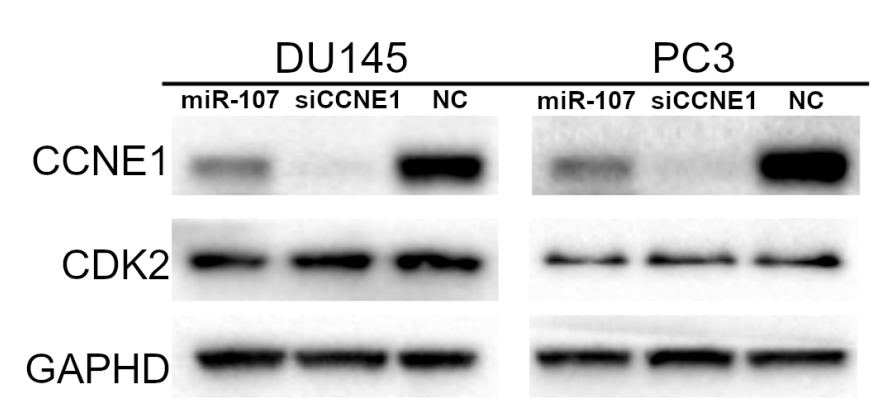

C
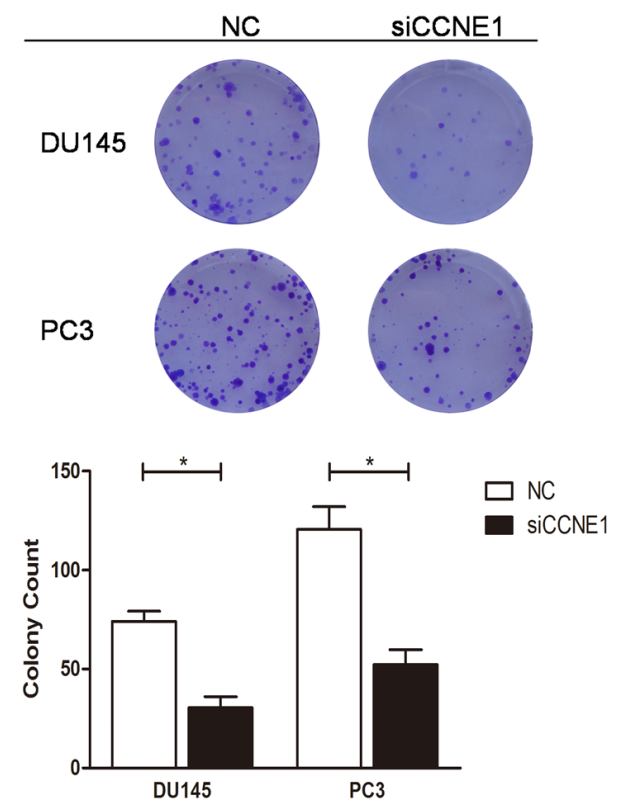

D

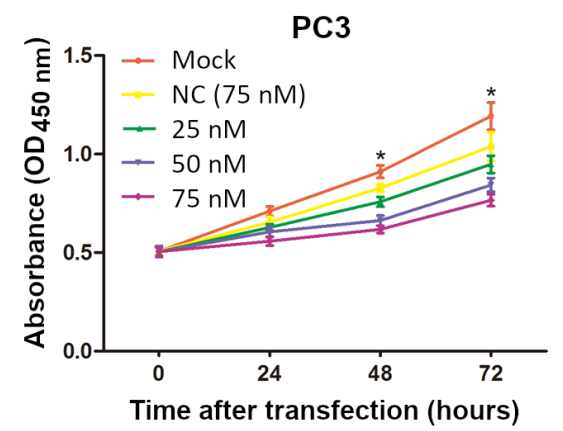

B
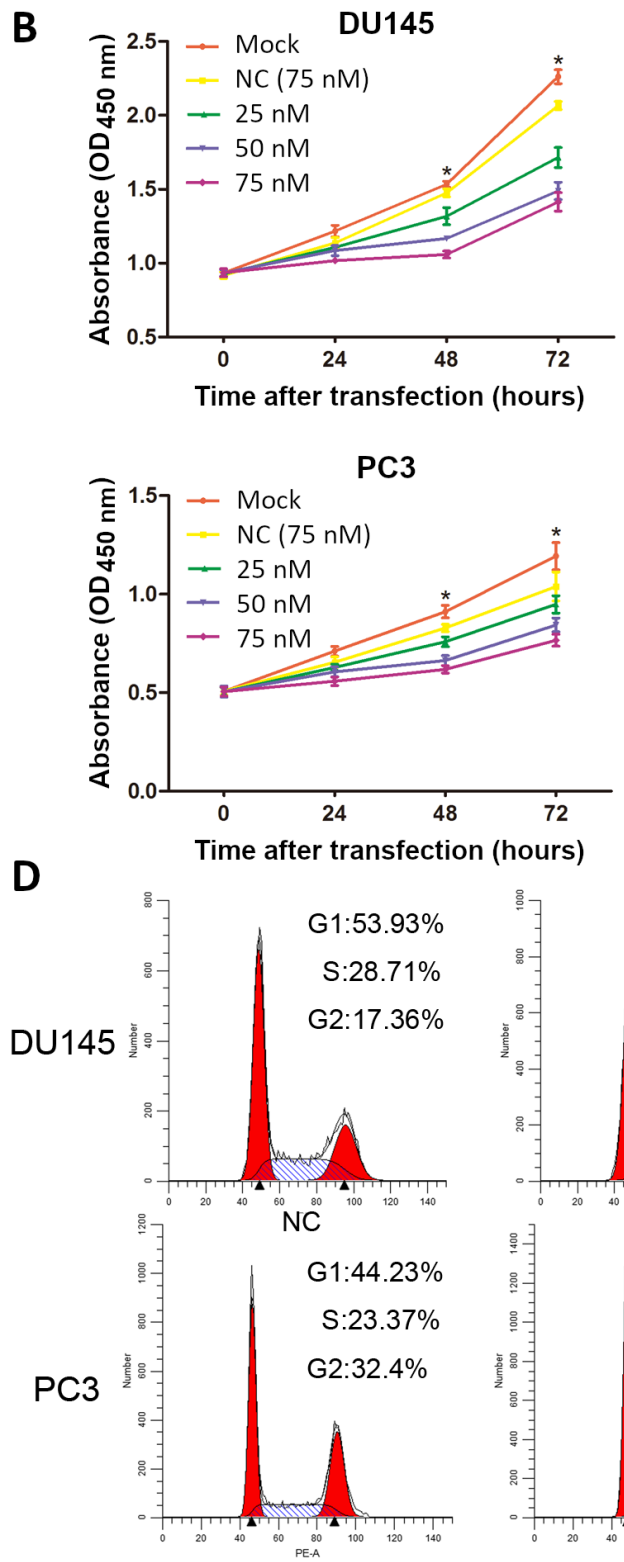
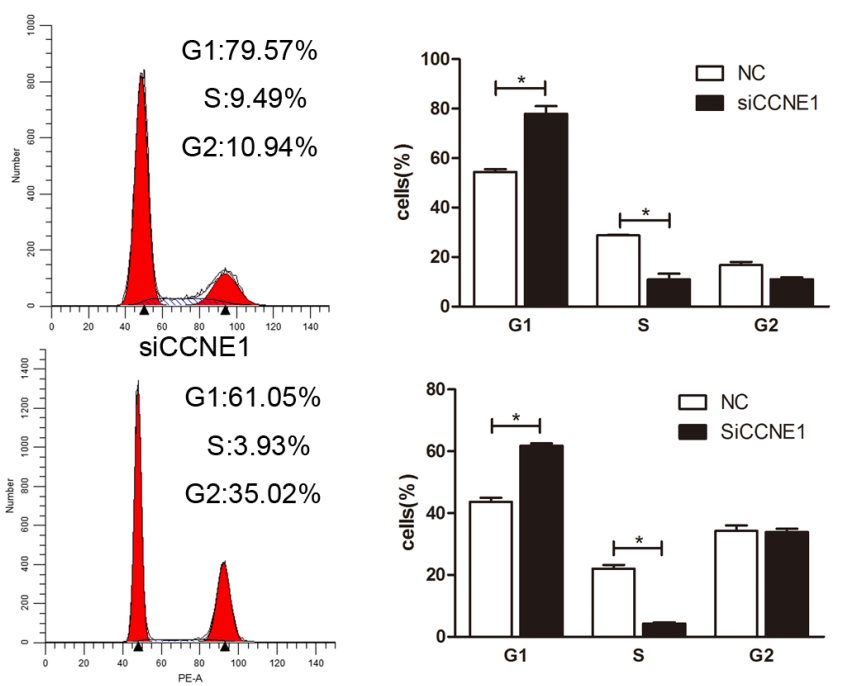

Figure 6. Knocking down of CCNE1 mimics the effects of miR-107 in PCa cells. A) Western blot analysis. CCNE1 siRNA knocked-down the expression of CCNE1 in PCa cells. But the CDK2 expression was not affected by miR-107 mimic transfection or CCNE1 knockdown. B) Growth curves of PCa cells transfected with CCNE1 siRNA in serial concentrations. CCNE1 siRNA-transfected PCa cells showed decreased viabilities compared with NC-treated PCa cells. Error bars represent S.D. from three independent experiments. C) Colony formation assay. The colony formation rates of PCa cells transfected with CCNE1 siRNA were reduced compared with NC-treated PCa cells. Error bars represent S.D. from three independent triplicate experiments. D) Flow cytometry cell cycle assay. Over-expression of CCNE1 siRNA induced G1/S arrest of PCa cells. Error bars represent S.D. from three independent experiments. ${ }^{*} \mathbf{p}<0.05$ 
(Figures 6B-6D). Further, intracellular co-transfection of miR-107 inhibitor could partially offset the knock-down of CCNE1 or the G1/S arrest induced by CCNE1 siRNA, with CCNE1 re-expressed (Figure 7A) and more cells re-entering into $S$ phase again (Figures 7B, 7C).

\section{Discussion}

Numerous miRNAs have been investigated with respect to their expression profiles in PCa. Those whose expression varies markedly between $\mathrm{PCa} /$ normal prostate cells

A

DU145

PC3

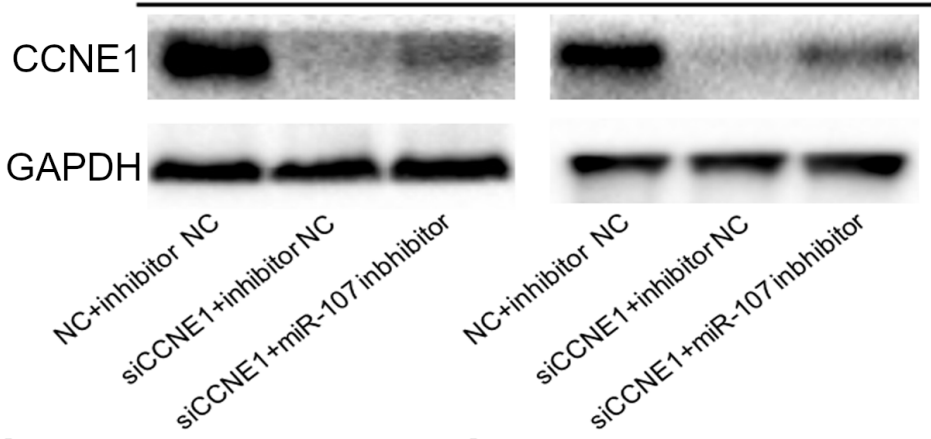

B
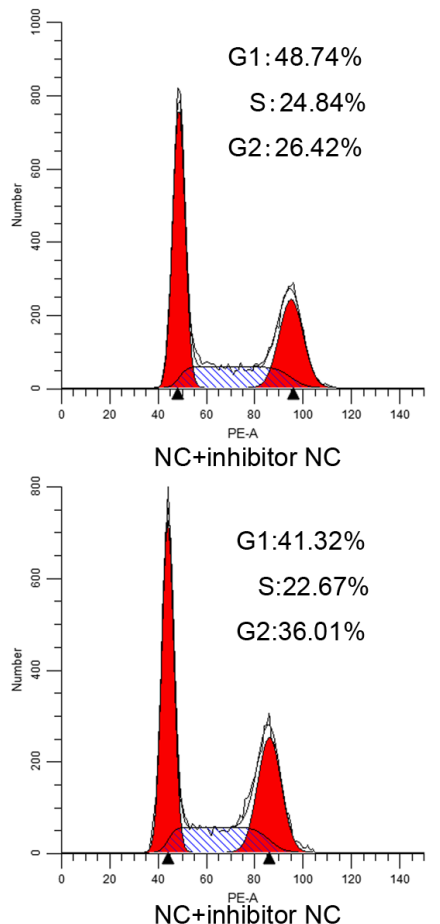

C

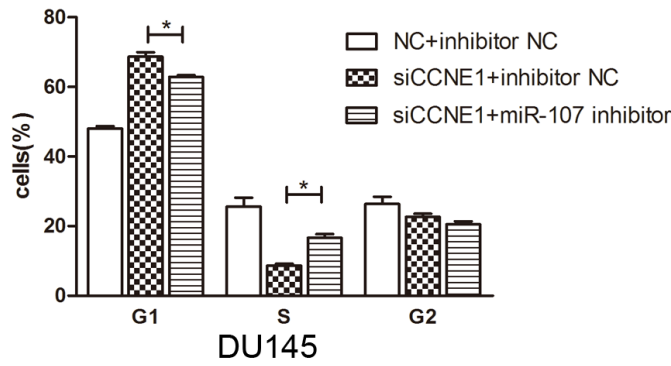

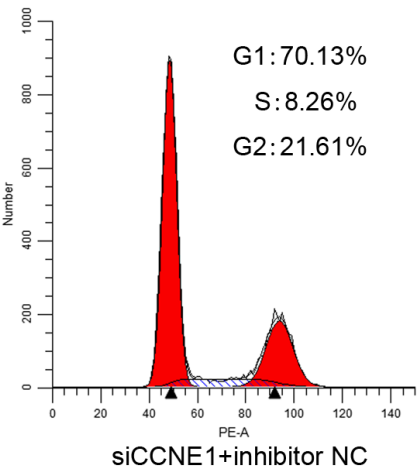

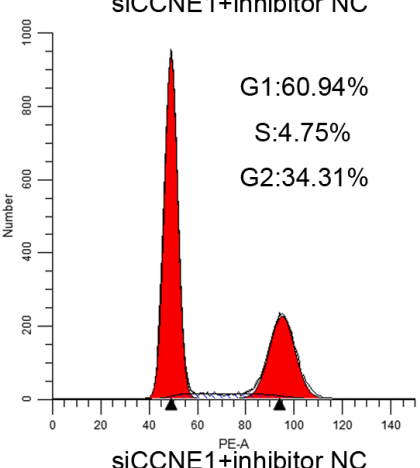

G1:62.39\%

S: $17.15 \%$

$\mathrm{G} 2: 20.46 \%$

DU145

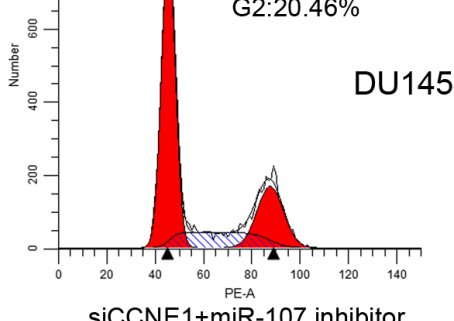

siCCNE1+miR-107 inhibitor

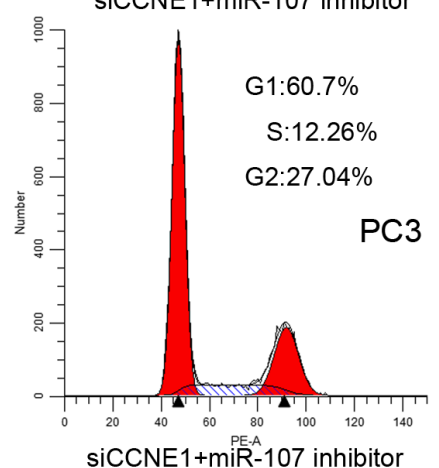

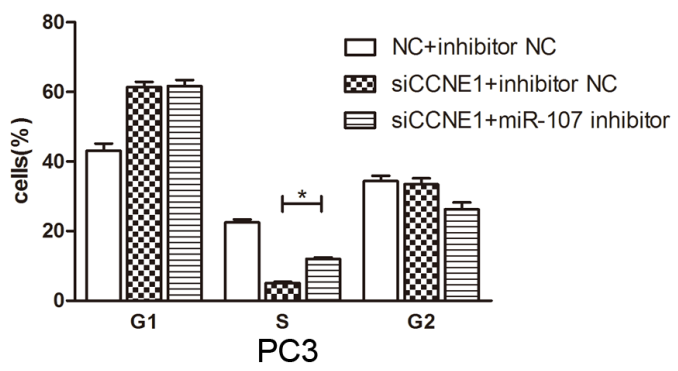

Figure 7. CCNE1 rescue experiments. A) Western blot analysis. Downregulation of miR-107 by miR-107 inhibitor reversed the expression of CCNE1 in CCNE1 knocked down PCa cells. B and C) Flow cytometry cell cycle assay. Downregulation of miR-107 by miR-107 inhibitor abolished the G1/S arrest caused by CCNE1 knock-down in PCa cells. Error bars represent S.D. from three independent experiments. ${ }^{*} \mathbf{p}<0.05$ 
and tissues have the potential to be either PCa biomarkers or therapeutic targets [19]. However, many of the miRNAs studied remain unexplored in terms of their actual biological functions in PCa. MiR-107 is an evolutionarily conserved miRNA, widely expressed across vertebrates. In the human genome, miR-107 gene resides within the introns of the gene of pantothenate kinase 1 (PANK1) [10], a rate-limiting enzyme in the synthesis of Coenzyme A [20-22]. In spite of the existing publications pertaining to the clinical values of miR-107 as a potential PCa biomarker in serum and urine $[23,24]$, the specific mechanisms by which miR-107 influences the tumorigenesis of PCa remain unknown.

In this research, for the first time, the functions of miR-107 in PCa were investigated systematically, and CCNE1 was identified as one of the direct targets of miR-107 in PCa cell line DU145 and PC3, as evidenced by qRT-PCR, western blot analysis, dual-luciferase reporter assay and the related cell function assays. The conclusion was also consolidated by the CCNE1 rescue experiments. CCNE1 is an oncogene commonly existing in human cells and is universally acknowledged to be crucial for cell cycle G1/S transition. In human, CCNE1 has been profiled and found to be ubiquitously amplified in many malignancies, such as ovarian cancer, endometrial carcinoma, breast cancer and osteosarcoma [25-29]. The importance of CCNE1 in the proliferation of PCa cells was also proved by this research, in which the cell viabilities and the colony-forming abilities of CCNE1 knocked-down $\mathrm{PCa}$ cells were significantly downregulated compared with NC-treated cells. CCNE1 exerts biological functions through its interaction with cyclin-dependent kinase 2 (CDK2). When CDK2 is bound by CCNE1, the ATP-binding pocket which is critical for CDK2 kinase activity opens up and allows access of both ATP and substrates to CDK2 catalytically active sites, and thus engenders serial downstream effects to induce $S$ phase entry [30-32]. This could explain our western blot results that the CDK2 expression was unaffected in miR-107 mimic-transfected or CCNE1 knocked-down PCa cells (Figure 6A), which corroborated that CCNE1 did not regulate CDK2 through the alteration of its quantities, for example transcriptional activation or inhibition, but through its conformational changes.

Compared with FISH miRNA assay, CISH allows simultaneous observation of histological and molecular alterations in a specimen [33]. Therefore, after the evaluation of miR-107 expression pattern in $\mathrm{PCa} /$ normal prostate cells, CISH experiment was conducted in PCa tissue microarray (TMA) to verify the expression pattern of miR-107 in PCa/peritumoral tissues. The results confirmed relatively low expression of miR-107 in the cytoplasm of PCa cells and tissues, in comparison with normal prostate cells and peritumoral tissues, which provided its antitumor role when overexpressed in PCa cell lines and revealed its diagnostic value in PCa. IHC staining of CCNE1 in PCa TMA also confirmed a relatively high expression of CCNE1 in PCa tissues in contrast to peritumoral tissues. However, being commercial- ized products, the TMAs did not contain information about the survival duration of PCa patients, hence there is need for analyses of the prognostic value of miR-107/CCNE1 in PCa based on more detailed information and larger sample size in the future.

Except for PCa, researchers have revealed the targets of miR-107 in multiple malignant tumors, such as breast cancer, cervical cancer, colorectal cancer and pancreatic ductal adenocarcinoma [34-37], where miR-107 either stimulated or inhibited proliferation and progression of these malignancies. The diversified tumorigenic or tumor-suppressive effects of miR-107 may seem contradictory, but given the idiosyncrasies of various human neoplasms, it is reasonable that the same miRNA could have different expression patterns, target different genes, and spawn different impacts in different cancers.

The fact that one microRNA can have diversified target genes could also explain the cell cycle changes induced by miR-107 overexpression in PC3 cells. Intracellular overexpression of miR-107 significantly increased the proportion of PC3 cells in G1 phase and decreased the proportion of PC3 cells in $\mathrm{G} 2$ phase, but did not change the S phase of PC3 cells (Figure 3A). This result didn't completely match with typical G1/S cell cycle arrest, however, it is possible that except for CCNE1, miR-107 could target other proteins in PC3 cells as well, and thus the typical G1/S cell cycle arrest induced by CCNE1 downregulation was scrambled.

It was also noted that the structural resemblance between miR-107 and miR-15/16/195/497 was of interest to some researchers, who considered them as one possible microRNA family, but no consensus has yet been reached on whether they exert identical effects [10]. Bonci et al. identified the direct targeting of BCL2 by miR-15/16 in PCa [38], however according to the findings in this work, miR-107 did not downregulate BCL2 expression (data not shown) or induce apoptosis in PCa cells. In fact, it has been reported that after glioneuronal H4 cells transfection with miR-15/16/107/195 respectively, the post-transfection mRNA profiling of $\mathrm{H} 4$ cells was not exactly the same, with approximately $20-50 \%$ differences among the down-regulated genes. This indicates incomplete overlaps of the target genes of miR-15/16/107/195 [10]. Therefore, despite the structural and functional overlaps of these microRNAs, it is still necessary to highlight their individual properties. In summary, the target genes of a particular miRNA (for example miR-107) cannot be simply extrapolated from its analogues, and it is essential to evaluate a specific miRNA in a specific physiological or pathological situation

In conclusion, miR-107 is downregulated in PCa cell lines and tissues and acts as a PCa tumor suppressor. It suppresses the proliferation and induces G1/S arrest of PCa cells by directly targeting the 3'-UTR of CCNE1. Notwithstanding the possibility that new target genes of miR-107 in PCa may be discovered in the future, this research provides the therapeutic potential of miR-107 in PCa. 
Supplementary information is available in the online version of the paper.

Acknowledgements: This study was supported by grants from National Natural Science Foundation of China (81772744, 81874203), and Zhejiang Provincial Natural Science Foundation of China (LY17H160019).

\section{References}

[1] BRAY F, FERLAY J, SOERJOMATARAM I, SIEGEL RL, TORRE LA et al. Global cancer statistics 2018: GLOBOCAN estimates of incidence and mortality worldwide for 36 cancers in 185 countries. CA Cancer J Clin 2018; 68: 394-424. https://doi.org/10.3322/caac.21492

[2] GREGORY RI, SHIEKHATTAR R. MicroRNA biogenesis and cancer. Cancer Res 2005; 65: 3509-3512. https://doi. org/10.1158/0008-5472.CAN-05-0298

[3] SOIFER HS, ROSSI JJ, SAETROM P. MicroRNAs in disease and potential therapeutic applications. Mol Ther 2007; 15: 2070-2079. https://doi.org/10.1038/sj.mt.6300311

[4] CULLIS PR, HOPE MJ. Lipid Nanoparticle Systems for Enabling Gene Therapies. Mol Ther 2017; 25: 1467-1475. https://doi.org/10.1016/j.ymthe.2017.03.013

[5] KULKARNI JA, CULLIS PR, VAN DER MEEL R. Lipid Nanoparticles Enabling Gene Therapies: From Concepts to Clinical Utility. Nucleic Acid Ther 2018; 28: 146-157. https:// doi.org/10.1089/nat.2018.0721

[6] HOY SM. Patisiran: First Global Approval. Drugs 2018; 78 : 1625-1631. https://doi.org/10.1007/s40265-018-0983-6

[7] RIZK M, TUZMEN S. Update on the clinical utility of an RNA interference-based treatment: focus on Patisiran. Pharmgenomics Pers Med 2017; 10: 267-278. https://doi. org/10.2147/PGPM.S87945

[8] WANG S, WANG X, LI J, MENG S, LIANG Z et al. c-Met, CREB1 and EGFR are involved in miR-493-5p inhibition of EMT via AKT/GSK-3beta/Snail signaling in prostate cancer. Oncotarget 2017; 8: 82303-82313. https://doi.org/10.18632/ oncotarget. 19398

[9] LI J, MENG S, XU M, WANG S, HE L et al. Downregulation of $\mathrm{N}(6)$-methyladenosine binding YTHDF2 protein mediated by miR-493-3p suppresses prostate cancer by elevating N(6)-methyladenosine levels. Oncotarget 2018; 9: 3752-3764. https://doi.org/10.18632/oncotarget.23365

[10] FINNERTY JR, WANG WX, HEBERT SS, WILFRED BR, MAO G et al. The miR-15/107 group of microRNA genes: evolutionary biology, cellular functions, and roles in human diseases. J Mol Biol 2010; 402: 491-509. https://doi. org/10.1016/j.jmb.2010.07.051

[11] BHATIA H, PATTNAIK BR, DATTA M. Inhibition of mitochondrial beta-oxidation by miR-107 promotes hepatic lipid accumulation and impairs glucose tolerance in vivo. Int J Obes (Lond) 2016; 40: 861-869. https://doi.org/10.1038/ ijo.2015.225

[12] CHEN L, LI ZY, XU SY, ZHANG XJ, ZHANG Y et al. Upregulation of miR-107 Inhibits Glioma Angiogenesis and VEGF Expression. Cell Mol Neurobiol 2016; 36: 113-120. https://doi.org/10.1007/s10571-015-0225-3
[13] FOLEY NH, O’NEILL LA. miR-107: a toll-like receptor-regulated miRNA dysregulated in obesity and type II diabetes. J Leukoc Biol 2012; 92: 521-527. https://doi.org/10.1189/ jlb.0312160

[14] WANG WX, RAJEEV BW, STROMBERG AJ, REN N, TANG $\mathrm{G}$ et al. The expression of microRNA miR-107 decreases early in Alzheimer's disease and may accelerate disease progression through regulation of beta-site amyloid precursor protein-cleaving enzyme 1. J Neurosci 2008; 28: 1213-1223. https://doi.org/10.1523/JNEUROSCI.5065-07.2008

[15] JIAO Y, KONG L, YAO Y, LI S, TAO Z et al. Osthole decreases beta amyloid levels through up-regulation of miR107 in Alzheimer's disease. Neuropharmacology 2016; 108: 332-344. https://doi.org/10.1016/j.neuropharm.2016.04.046

[16] STONE KR, MICKEY DD, WUNDERLI H, MICKEY GH, PAULSON DF. Isolation of a human prostate carcinoma cell line (DU 145). Int J Cancer 1978; 21: 274-281. https://doi. org/10.1002/ijc.2910210305

[17] KAIGHN ME, NARAYAN KS, OHNUKI Y, LECHNER JF, JONES LW. Establishment and characterization of a human prostaticcarcinomacellline(PC-3).InvestUrol1979;17:16-23.

[18] WEBBER MM, BELLO D, QUADER S. Immortalized and tumorigenic adult human prostatic epithelial cell lines: characteristics and applications. Part I. Cell markers and immortalized nontumorigenic cell lines. Prostate 1996; 29: 386-394. https://doi.org/10.1002/(SICI)10970045(199612)29:6<386::AID-PROS7>3.0.CO;2-6

[19] KUMAR B, LUPOLD SE. MicroRNA expression and function in prostate cancer: a review of current knowledge and opportunities for discovery. Asian J Androl 2016; 18: 559567. https://doi.org/10.4103/1008-682X.177839

[20] ROCK CO, CALDER RB, KARIM MA, JACKOWSKI S. Pantothenate kinase regulation of the intracellular concentration of coenzyme A. J Biol Chem 2000; 275: 1377-1383. https://doi.org/10.1074/jbc.275.2.1377

[21] RAMASWAMY G, KARIM MA, MURTI KG, JACKOWSKI S. PPARalpha controls the intracellular coenzyme A concentration via regulation of PANK1alpha gene expression. J Lipid Res 2004; 45: 17-31. https://doi.org/10.1194/jlr.M300279JLR200

[22] DE VILLIERS M, BARNARD L, KOEKEMOER L, SNOEP JL, STRAUSS E. Variation in pantothenate kinase type determines the pantothenamide mode of action and impacts on coenzyme A salvage biosynthesis. FEBS J 2014: 281: 47314753. https://doi.org/10.1111/febs.13013

[23] LODES MJ, CARABALLO M, SUCIU D, MUNRO S, KUMAR A et al. Detection of cancer with serum miRNAs on an oligonucleotide microarray. PLoS One 2009; 4: e6229. https://doi.org/10.1371/journal.pone.0006229

[24] BRYANT RJ, PAWLOWSKI T, CATTO JW, MARSDEN G, VESSELLA RL et al. Changes in circulating microRNA levels associated with prostate cancer. Br J Cancer 2012; 106: 768-774. https://doi.org/10.1038/bjc.2011.595

[25] GOUNDIAM O, GESTRAUD P, POPOVA T, DE LA MOTTE ROUGE T, FOURCHOTTE V et al. Histo-genomic stratification reveals the frequent amplification/overexpression of CCNE1 and BRD4 genes in non-BRCAness high grade ovarian carcinoma. Int J Cancer 2015; 137: 1890-1900. https://doi.org/10.1002/ijc.2956 
[26] KANSKA J, ZAKHOUR M, TAYLOR-HARDING B, KARLAN BY, WIEDEMEYER WR. Cyclin E as a potential therapeutic target in high grade serous ovarian cancer. Gynecol Oncol 2016; 143: 152-158. https://doi.org/10.1016/j.ygyno.2016.07.111

[27] NAKAYAMA K, RAHMAN MT, RAHMAN M, NAKAMURA K, ISHIKAWA $M$ et al. CCNE1 amplification is associated with aggressive potential in endometrioid endometrial carcinomas. Int J Oncol 2016; 48: 506-516. https://doi. org/10.3892/ijo.2015.3268

[28] LODEN M, STIGHALL M, NIELSEN NH, ROOS G, EMDIN SO et al. The cyclin D1 high and cyclin E high subgroups of breast cancer: separate pathways in tumorogenesis based on pattern of genetic aberrations and inactivation of the pRb node. Oncogene 2002; 21: 4680-4690. https://doi. org/10.1038/sj.onc.1205578

[29] LOCKWOOD WW, STACK D, MORRIS T, GREHAN D, O'KEANE C et al. Cyclin E1 is amplified and overexpressed in osteosarcoma. J Mol Diagn 2011; 13: 289-296. https://doi. org/10.1016/j.jmoldx.2010.11.020

[30] JEFFREY PD, RUSSO AA, POLYAK K, GIBBS E, HURWITZ J et al. Mechanism of CDK activation revealed by the structure of a cyclinA-CDK2 complex. Nature 1995; 376: 313-320. https://doi.org/10.1038/376313a0

[31] SATYANARAYANA A, KALDIS P. Mammalian cell-cycle regulation: several Cdks, numerous cyclins and diverse compensatory mechanisms. Oncogene 2009; 28: 2925-2939. https://doi.org/10.1038/onc.2009.170

[32] PELLERANO M, TCHERNIUK S, PERALS C, NGOC VAN TN, GARCIN E et al. Targeting Conformational Activation of CDK2 Kinase. Biotechnol J 2017; 12. https://doi. org/10.1002/biot.201600531
[33] MARDEKIAN SK, SOLOMIDES CC, GONG JZ, PEIPER SC, WANG ZX et al. Comparison of Chromogenic In Situ Hybridization and Fluorescence In Situ Hybridization for the Evaluation of MDM2 Amplification in Adipocytic Tumors. J Clin Lab Anal 2015; 29: 462-468. https://doi.org/10.1002/ jcla.21794

[34] ZHANG L, MA P, SUN LM, HAN YC, LI BL et al. MiR-107 down-regulates SIAH1 expression in human breast cancer cells and silencing of miR-107 inhibits tumor growth in a nude mouse model of triple-negative breast cancer. Mol Carcinog 2016; 55: 768-777. https://doi.org/10.1002/mc.22320

[35] ZHOU C, LI G, ZHOU J, HAN N, LIU Z et al. miR-107 activates ATR/Chk1 pathway and suppress cervical cancer invasion by targeting MCL1. PLoS One 2014; 9: e111860. https:// doi.org/10.1371/journal.pone.0111860

[36] MOLINA-PINELO S, CARNERO A, RIVERA F, ESTEVEZ-GARCIA P, BOZADA JM et al. MiR-107 and miR-99a$3 p$ predict chemotherapy response in patients with advanced colorectal cancer. BMC Cancer 2014; 14: 656. https://doi. org/10.1186/1471-2407-14-656

[37] XIONG J, WANG D, WEI A, LU H, TAN C et al. Deregulated expression of miR-107 inhibits metastasis of PDAC through inhibition PI3K/Akt signaling via caveolin-1 and PTEN. Exp Cell Res 2017; 361: 316-323. https://doi.org/10.1016/j. yexcr.2017.10.033

[38] BONCI D, COPPOLA V, MUSUMECI M, ADDARIO A, GIUFFRIDA $\mathrm{R}$ et al. The miR-15a-miR-16-1 cluster controls prostate cancer by targeting multiple oncogenic activities. Nat Med 2008; 14: 1271-1277. https://doi.org/10.1038/ nm. 1880 